\title{
ТЕОРЕТИЧЕСКИЕ ОСНОВЫ МОДЕЛЬНОГО И КОНСТРУКТИВНОГО УПРАВЛЕНИЯ ЦИФРОВОЙ ЭКОНОМИКОЙ
}

\author{
(c) 2019 Волков Денис Владимирович \\ Старший инвестиционный менеджер инвестиционной компании ООО «ДИ»
}

В статье акцентируется внимание на управлении цифровой экономикой как макросистемой, роли модельно-конструктивных инструментов, экономико-математических методов и новейших вычислительно-информационных средств в повышении эффективности этого управления, определении важнейших направлений цифроэкономики в современной России.

Ключевые слова: промышленная революция, макросистема, цифроэкономика, управление цифроэкономикой, модельно-конструктивные инструменты управления, экономическое моделирование.

С появлением цифры (знака), счёта человек стал жить в цифровой реальности и, следовательно, его экономика, т.е. особая сфера общественной жизни, в которой он осуществлял хозяйственную деятельность, всегда требовала сопоставления затрат и результатов. Во все времена это непременное математическое сопоставление, составляющее, по четкому выражению Ф. Энгельса, «пространственные формы и количественные отношения действительного мира» позволяло и позволяет хозяйствующим субъектам определять результативность (эффективность) своего хозяйствования. Следовательно, экономика всегда была цифровой и такой она, по-видимому, и останется, дополняясь всё новыми счётными возможностям и вычислительными средствами.

Считается, что первые цифры появились у египтян и вавилонян. У ряда народов (древние греки, финикяне, евреи, сирийцы) ими служили буквы алфавита. Подобная система применялась и в России вплоть до 16 столетия. В Средние века в Европе пользовались системой римских цифр, основанной на употреблении особых знаков для десятичных разрядов I=1, X=10, C=100, M=1000 и их половин $\mathrm{V}=5, \mathrm{~L}=50, \mathrm{D}=500$. Современные цифры (арабские) перенесены в Европу в 13 веке (по всей вероятности, из Индии) и получили широкое распространение со второй половины 15 века. С использованием цифр связано исчисление (нумерация) как способ выражения и обозначения чисел. В системах исчисления некоторое число $\mathbf{n}$ единиц (к примеру, 10) обозначаются в одну единицу 2-го разряда (десяток), то же число единиц 2-го разряда объединяется в единицу 3-го разряда (сотню) и т.д. Число $\mathbf{n}$ считается основанием системы исчисления, а знаки, употребляемые для обозначения количества единиц каждого разряда,- цифрами.

С развитием производительных сил общества (средств труда, предметов труда и рабочей силы) потребовалась модификация монетарных инструментов управления экономикой, главенствующих в условиях господства товарно-денежных отношений. И если до 21-го столетия такими инструментами были (и пока остаются) экономические нормативы, различного рода ограничения, правила, налоги, платежи, штрафы, санкции, цены, вознаграждения, стимулы, кредиты, инвестиции, субсидии, дотации, трансферты, заказы, преференции и др., то со второго его десятилетия стали особенно бурно развиваться технологии, которые существенно изменяют производство, меняют жизнь людей, и вместе с тем суть управления общественно-экономическими процессами.

Автор статьи принял участие в НИР «Анализ международной системы электронного обмена деловой и коммерческой информацией и разработка мероприятий по ее использованию в РФ», проведенной под научным руководством д.э.н., проф., И.М. Братищева. Выяснилось, к примеру, что новая цифровая реальность стала проявляться уже тогда, когда возникла кибернетика как наука об информации и управлении. Бум цифровизации произошел после того, когда в мире заговорили о новой промышленной (производственной) революции 21 века. Толчком стала книга американского экономиста Джереми Рифкина «Третья промышленная революция» [1], засвидетельствовавшая появление интегрированных технологий, развивающихся на базе искусственного интеллекта, где ведущая роль стала отводиться Интернету вещей, робототехнике, созданию новых материалов с заданными свойствами. 
Следует отметить, что нестандартные ответы на нестандартные вопросы, которые ставит 21 век, дает в своей книге С.В. Хапров. Он утверждает, что по миру уже распространяется цифровой коммунизм, коренным образом меняющий экономику, образование, транспорт и даже отношение к сырьевым компаниям, и высказывает предположение, что наступающая эпоха потенциально более выгодна именно русской цивилизации [2].

Анализу особенностей четвертой промышленной революции посвящена монография Е.Б. Липкина «Индустрия 4.0: Умные технологии - ключевой элемент в промышленной конкуренции» [3]. В ней анализируются предпосылки системных изменений в промышленных технологиях, рассматривается технологическая основа новой промышленной революции, исследуются направления изменений, происходящих в ходе этой революции (тотальная цифровизация, развитие аналитических систем, умные продуктово-сервисные системы, логистика 4.0, умное производственное оборудование), определяется эффект от применения новых технологий, раскрываются преграды на пути технического прогресса, такие как: консерватизм, необходимость инвестиций, устаревшие производственные фонды, недоверие к машинам, безопасность данных, правовые вопросы, стандарты, отсутствие профильных специалистов и другие проблемы.

Журнал «Изборский клуб» в конце 2017 года издал номер, названный «Цифровая экономика. Виртуальность и реальность» [4]. В нем содержится доклад Изборскому клубу академика РАН С.Ю. Глазьева «Информационно-цифровая революция в контексте структурных изменений в экономике», материалы круглого стола Изборского клуба «Цифровая мобилизация», статья В.В. Иванова и Г.Г. Малинецкого «Цифровая экономика: мифы, реальность, перспективы», А.И. Агеева «Цифровизация как цивилизационный феномен», статьи других авторов.

Во всех перечисленных публикациях содержится глубокий анализ цифровой экономики, подчеркивается, что человечество переходит в мир, где компьютеры и алгоритмы могут анализировать (выявлять ранее скрытые шаблоны), оптимизировать, прогнозировать, настраивать (задавать параметры какого-либо продукта или услуги), оцифровывать и автоматизировать все больше и больше производственных процессов.
Любой хозяйствующий субъект (компания, корпорация), которая не задействует все перечисленные выше элементы столкнется с большими трудностями, и это меняет каждый вид деятельности в реальном секторе экономики.

Что необходимо предпринимать, когда на планете, скажем, меняется климат? Необходима адаптация к этим изменениям с тем, чтобы смягчить последствия ухудшения природно-климатических условий и получить максимум возможных выгод от этих изменений. Понятно, что такая адаптация должна произойти на всех уровнях жизнедеятельности человека: индивидуальном, коллективном и национальном. Так и в экономке. Ее цифровизация требует не только адаптации людей и общества в целом к новым реалиям, но и актуализирует проблему управления цифровой экономикой, как экономикой «функционирующей в условиях полной информационной транспарентности, т.е. прозрачности, где каждое действие экономических субъектов фиксируется, проверяется и запоминается и где постоянно действуют обратные связи». K этому следует добавить, что цифроэкономика базируется на новой цифровой реальности:

- больших данных (big data);

- нейротехнологиях и искусственном интеллекте;

- системе распределенного реестра (списка, перечня);

- квантовых, т.е. микротехнологиях;

- новых производственных технологиях;

- промышленном интернете;

- компонентах робототехники и сенсорики;

- технологиях беспроводной связи;

- технологиях виртуальной и дополненной реальности.

Все эти составляющие, как считают аналитики, входят в технологическую основу «Индустрии 4.0» И «Индустрии 5.0», которые в последнее время стали предметом внимания западных и наших отечественных ученых, исследующих проблемы становления и укрепления шестого технологического уклада (ТУ6). Этот уклад характеризуется уже не энергией воды (ТУ1), пара (ТУ2), электричества (ТУ3), углеводородов (ТУ4), информации (ТУ5), а энергией частиц, энергией ансамблей. Главным образом, поэтому он актуализирует потребность в новых формах хозяйствования, основанных на цифровых трансформациях, а также требует перехода к неоцифросфере, как новой цивилизационной, 
философской и экономической реальности. В этих условиях возникает потребность:

во-первых, в «больших данных», обязательно очищенных от повторного счета, а, следовательно, достоверных и полученных в реальном времени;

во-вторых, в совокупности алгоритмов, т.е. систем вычислений, направленных на решение тех или иных технико-экономических задач;

в-третьих, в создании новых коммуникационных сетей (человеко-машинных интерфейсов), дающих хозяйствующим субъектам возможность взаимодействия между собой и объектами других уровней;

в-четвертых, в новых организационно-правовых формах хозяйствующих субъектов и их объединений (корпораций).

Следовательно, сама логика развития производительных сил общества на основе новейших достижений науки и техники определяет необходимость, потребность и возможность управления экономикой как макросистемой при помощи не только традиционных, но и модельно-конструктивных инструментов, предполагающих использование современных экономико-математических методов и новейших вычислительно-информационных средств. Это предполагает осуществление интегрированного прогнозирования и мобилизационного проектирования в целях достижения самодостаточности и устойчивости развития российской экономики.

При этом следует учитывать, что цифровая экономика это уже не классическая и даже не постклассическая рыночная экономика, в которой обмен деятельностью и ее результатами осуществляется посредством использования механизма товарно-денежных отношений. Представляется, что управление такой новой экономикой потребует активизации использования экономико-математических методов, что позволит получать численные результаты на базе прогнозной, статистической и онлайн-информации.

Управление с экономической точки зрения есть сознательное целенаправленное воздействие со стороны субъектов, руководящих органов на людей (в т.ч. и совокупных работников) и экономические объекты, осуществляемое с целью направить их действия на получение же- лаемых экономических результатов.

Мы же ведем речь о необходимости дополнить эти методы модельно-конструктивными инструментами". С методологической точки зрения такое управление требует определения критериев (отличительных признаков) и его научности. На наш взгляд, такими критериями могут быть:

во-первых, четкое формулирование аксиом, определений, описывающих экономические реалии. Наличие логических умозаключений (дедукции), ведущих от общего к частному и математических преобразований, позволяющих выявить следствия из исходных данных;

во-вторых, конкретизация интуитивных представлений о том или ином экономическом процессе в виде индуктивного наведения, играющего большую роль в формулировании математических моделей, характеризующих взаимосвязь между процессами и явлениями экономической действительности;

в-третьих, практическая проверка полученных результатов путем проведения, к примеру, экономических экспериментов. При этом следует учитывать, что, как правило, в математической литературе во внимание зачастую принимается лишь комплекс условий, характерный для активно воспроизводимого эксперимента. В реальных же задачах пассивного эксперимента выяснение этих условий может быть осуществлено посредством математического анализа.

K сожалению, произошедшие в современной России так называемые «рыночные преобразования» протекали в условиях дезинформации в различных областях знаний, в том числе и главным образом в экономической науке. По всей вероятности, именно это обусловило перманентное разрушение материального производства, духовной культуры, депопуляцию населения (в основном проживающего в средней полосе нашей страны, в Сибири и на Дальнем востоке). Дезинформация (в том числе и в научной сфере) препятствует выработке социально и экономически значимых вариантов преодоления сложившегося положения. Зачастую она может принимать вид математизированного наукообразия, объективизации тех или иных ложных в своей сути построений, как бы прикрываемых объективными законами, и мешает перспективной выработке приемлемого для

\footnotetext{
* В данном случае под моделью (лат. modulus) понимается мера, образец, а под конструктивным (лат. constructio) - составление, построение.
} 
России варианта развития.

Предлагаемый нами модельно-конструктивный подход, ограждая науку от наукообразия, облегчает такую выработку, поскольку уже существующих ограничений в виде логики (формальной и диалектической), математической логики и различных доктринальных проектов, как свидетельствует современная практика, для этих целей недостаточно. К тому же опыт свидетельствует, что практическая реализация либеральных доктрин России, как и во многих других странах, слишком дорого обходится людям. С использованием же модельно-конструктивных инструментов возможно не только прояснить перспективы развития отечественной экономики, причины ее сегодняшнего неудовлетворительного состояния, но и исследовать вызовы и угрозы ее цифровизации, которые следовало бы учитывать в национальной программе «Цифровая экономика Российской Федерации».

В силу вышеизложенного, возникает вполне правомерный вопрос о том, что представляют собой модельно-конструктивные инструменты управления экономикой в ее цифровой ипостаси? Необходимо отметить, что метод моделирования в экономике для исследования экономических систем посредством создания их абстрактного образца, как правило, отражает главные соотношения и основные черты описываемых моделью экономических явлений и процессов, абстрагируясь от второстепенных моментов. Следовательно, модели в экономике не могут быть полностью адекватными моделируемому объекту. Наиболее широко экономическое моделирование стало использоваться со второй трети 20 века после возникновения таких новых областей математики, как «исследование операций» и после создания ЭВМ, способных решать сложные задачи. Именно тогда, а не в нулевые годы 21 века, как принято считать, и возникла собственно цифровая экономика как «экономика цифрового мира», в котором сделки и транзакции стали осуществляться алгоритмически, т.е. по определенным правилам, на основе анализа «больших данных».

Процесс использования модельно-конструктивных инструментов в управлении цифроэкономикой условно можно подразделить на следующие этапы. Первый этап: конструирование (формулирование) общей задачи. Это предполагает определение объекта исследования: экономика страны, отрасли, предприятия, кор- порации, фирмы, либо определенного аспекта функционирования экономической системы (производство, распределение, обмен, потребление), т.е. ее расширенного воспроизводства. Затем формулируются требования к характеру исходной информации, которая может быть статистической, получаемой в результате наблюдений за экономическими процессами, или нормативной (коэффициенты затрат на производство, научно-обоснованные нормы потребления и т.д.). И, наконец, изучаются исходные (простейшие) свойства моделируемого объекта и вместе с тем выдвигаются гипотезы о характере его развития, которые, как правило, многовариантны. Таким образом, вся информация, получаемая на первом этапе, приобретает форму совокупности исходных предпосылок собственно моделирования системы.

Содержанием второго этапа является создание модели той или иной экономической системы. Заметим, модели, используемые в целях управления экономическими процессами могут быть самыми разными. Так, наиболее общие закономерности развития экономики исследуются, а затем могут использоваться в управлении, с помощью, так называемых балансовых, оптимизационных, равновесных, игровых и др. моделей. Анализировать и прогнозировать динамику и соотношение различных синтетических показателей, таких как ВВП, занятость, сбережения, инвестиции и т.д. можно, используя макроэкономические модели, скажем, производства, обмена (торговли), снабжения и сбыта. Считается, что наиболее пригодными для исследования сложных экономических систем являются математические модели. Для анализа простейших экономических процессов (в частности, на транспорте) применяются аналоговые модели. Начиная с 60-х годов прошлого века, большую популярность приобрели имитационные модели. Они позволяют изучать реальные процессы экономических систем, в частности, в тех случаях, когда их математический анализ затруднен или даже невозможен. То есть имитационное моделирование заменяет собой экспериментальное исследование экономических систем. Следует отметить, что классификация (в известном смысле условная) экономических моделей осуществляется по следующим основным критериям: целям и задачам, объекту, используемому аппарату исследования, характеру исходной информации (с точки зрения этого 
критерия различаются статистические и нормативные модели).

Важно иметь в виду, что на этапе построения математической модели результаты эмпирических исследований переводятся со специфического экономического языка на универсальный математический язык, выбирается схема, конструкция модели, вводятся переменные, параметры и функциональные зависимости. При этом построение моделей, свойства которых еще не изучены (в нашем случае это модельно-конструктивный инструмент управления цифроэкономикой) стимулирует развитие самой математики и появление ее новых направлений.

На третьем этапе осуществляется математический анализ сформулированной модели, который предполагает получение не только качественных, но и количественных выводов, которые детализируют круг явлений, входящих в пределы данной модели. Очень важно иметь ввиду, что качественные выводы, которые можно получить анализируя сформулированную модель, позволяют обнаружить неизвестные ранее свойства экономической системы, весьма важные с управленческой точки зрения. К их числу могут относиться: структура системы, динамика ее развития, устойчивость, соотношение макроэкономических параметров, свойства ценностных показателей и т.п. К примеру, на основе так называемых моделей сбалансированного роста ученым удалось выяснить асимптоматичные свойства эффективных экономических траекторий к стационарному развитию с максимальным темпом, с помощью моделей оптимального планирования исследовать теоретические проблемы ценообразования.

Что касается количественных выводов из экономических моделей, то к ним относятся оптимальные планы развития тех или иных хозяйственных структур, прогнозы экономической динамики, расчеты цен и т.п. Такого рода действия дают возможность достигать большого экономического эффекта.

Модельно-конструктивные инструменты, используемые наряду с монетарными инструментами, являются важнейшими (если не самыми важными) элементами автоматизированных систем управления, основанных на новейших достижениях аппаратного и программного сопровождения, а также на современных информационных технологиях. K примеру, грандиозным проектом 20 века стало создание в Советском Союзе АСУ (А+9+1000), т.е. автоматизированной системы управления девятью отраслями экономики с включенными в них тысячью промышленных предприятий ВПК. В рамках этого проекта были созданы:

- автоматизированная система цехового управления предприятия (АСУ-цех);

- АСУ главного производственного управления;

- Автоматизированные рабочие места специалистов (АРМ).

Наряду с этим в СССР была введена система «Алмаз», включившая министерства, крупные объединения и заводы, осуществляющие реализацию решений Госплана в реальном масштабе времени, а также государственный фонд алгоритмов и программ. Однако в 90-е годы прошлого века развитие этого проекта не последовало. Действия «реформаторов» были направлены на атомизацию производства и всей экономики, что сопровождалось изменением экономической и мировоззренческой оценки электроники. Наряду с IT-технологиями и информатикой они были отнесены к сфере услуг. Но можно ли с этим согласиться?

Управляемая посредством модельно-конструктивных инструментов цифровая экономика, по мнению специалистов, меняет фундаментальную основу экономической и финансовой «матрицы» общества, порождает потребность в системных технологических прорывах и новых способах обработки информации. Она создает условия не только для оцифровывания всего или почти всего, но и для целенаправленного (в том числе экспериментального) управления социально-экономическими процессами путем консолидации информационно-вычислительных систем и их быстродействия, позволяющего оптимизировать производство и, в известном смысле, потребление, проектирование новых продуктов, а также любых видов массовых, групповых и индивидуальных сознаний посредством адаптации многих полезных функций жизнеобеспечения, подчиняющихся алгоритмизации (так называемые «беспилотные системы»).

Поскольку модели могут быть самыми разными, постольку и конструктивные требования к ним различны. Так, теоретические модели имеют абстрактный вид, поскольку они отображают лишь самые общие свойства экономических цифровых систем. В свою очередь, с помощью математических методов доказывается 
необходимость достижения эффективного (равновесного, оптимального) состояния (траектории развития) экономической системы, т.е. национальной экономики в целом, ее отраслей и кластеров, отдельных корпораций и хозяйственных единиц и по возможности строится алгоритм достижения такого состояния. Практика свидетельствует, что алгоритм решения любой экономической задачи - это зачастую отображение процессов, реально протекающих в моделируемом объекте. Модели, применяемые для конкретных расчетов, в качестве своей теоретической основы имеют абстрактный вид и выступают результатом анализа последних.

В любом случае конкретные модели, используемые в качестве инструментов управления цифросферой экономики, способны достаточно полно отражать специфику исследуемого экономического объекта, и именно это обстоятельство дает возможность использовать расчеты, осуществляемые в ходе моделирования, в практических целях, т.е. осуществлять конкретно экономическую интерпретацию полученных результатов.

Таким образом, моделирование экономических процессов в целях управления цифроэкономикой позволяет переводить математические понятия на язык изучаемого объекта, интерпретировать качественные результаты как свойства и закономерность развития экономических систем, а сформулированный при этом алгоритм становится инструментом управления, тогда как числовые результаты конкретным планом или прогнозом.

Перед тем как использовать полученные теоретические и практические выводы необходимо осуществить четвертый этап формирования модельно-конструктивных инструментов для управления цифроэкономикой, который состоит в проверке полученных результатов. Дело это не простое, поскольку обычные способы естественных наук - эксперимент, сопоставление полученных результатов с характеристиками реальных процессов и др. в экономике могут применяться не всегда. К примеру, если программа развития того или иного структурного элемента экономической системы, созданного посредством экономического моделирования отражает необходимость совершенствования практики, то еще не факт, что эта необходимость вызвана несовершенством именно управления. Вполне возможно, что в исходной модели не были учтены некоторые условия, имеющие место в реальной действительности и, следовательно, намеченные с помощью моделирования улучшения не могут быть осуществлены. Поэтому теоретическая проверка правильности исходных предпосылок чрезвычайно важна. Тем более, что экономический эксперимент, на конкретном объекте или аналоговом устройстве, имитирующем ту или иную модель (например, модель динамического межотраслевого баланса или модель развивающейся экономики), либо эксперимент, дающий возможность проверить результаты моделирования, требует серьезных затрат, а эксперимент, проводимый на конкретном объекте всегда вызывает трудности социально-экономического характера.

Наконец, последний пятый этап (внедрение) предполагает, разумеется, в случае положительного завершения предыдущего этапа, совершенствование как самой экономической теории, так и методов управления цифровой экономикой. В том случае, если это не достигается, следует уточнять исходные предпосылки модели, т.е. вновь пройти все этапы моделирования.

Таким образом, цифровая экономика - это такой тип экономики, в котором сделки и транзакции, как уже отмечалось, осуществляются алгоритмически, т.е. посредством математической системы операций (вычислений), применяемых последовательно по строго определенным правилам и на основе интеллектуального анализа больших данных, открывает принципиально новые возможности использования модельно-конструктивных инструментов в управлении. Вместе с тем, нельзя не учитывать, что эти инструменты имеют определенные границы использования, не вся информация об экономических процессах может быть формализована и потому не вся может быть доступной и поддающейся теоретическому анализу. Очевидно, что даже самые современные вычислительные средства не могут справиться с тем громадным объемом вычислений, которые требуется осуществить для решения конкретных экономических задач. Поэтому модельно-конструктивные инструменты используемые в управлении цифровой экономикой, необходимо дополнять другими. Одним из них может быть так называемый «метод сумм времени», в свое время разработанный немецкими учеными. Его, к примеру, можно использовать в разноуровневом планировании, логистике и др. сферах. K сожалению, этот метод, по всей вероятности, в силу его сложности так и не получил воплощения в жизнь. 


\section{Библиографический список}

1. Рифкин Д. Третья промышленная революция. Москва. 2014. С. 510.

2. Хапров С.В. Цифровой коммунизм. Москва. 2013. С. 181.

3. Липкин Е.Б. Индустрия 4.0: Умные технологии - ключевой элемент в промышленной конкуренции. Москва. 2017. 2017. С. 224.

4. Глазьев С.Ю., Иванов В., Малинецкий Г., Сундиев И., Агеев А., Анпилогов А. // Изборский клуб. Общественно-политический журнал. № 8 (54). 2017. С. 12-98.

5. «Цифровая экономика Российской Федерации». Программа, утвержденная Правительством РФ 28 июля 2017 года. (http://static.government.ru/media/files/9gFM4FHj4PsB79I5v7yLVuPgu4bvR7M0.pdf).

Поступила в редакцию 28.02.2019 